\title{
BMJ open Cystatin C in a composite risk score for mortality in patients with infective endocarditis: a cohort study
}

\author{
Christian Bjurman, ${ }^{1}$ Ulrika Snygg-Martin, ${ }^{2}$ Lars Olaison, ${ }^{2}$ Michael L X Fu, ${ }^{1}$ \\ Ola Hammarsten ${ }^{3}$
}

To cite: Bjurman C, Snygg-Martin U, Olaison L, et al. Cystatin $\mathrm{C}$ in a composite risk score for mortality in patients with infective endocarditis: a cohort study. BMJ Open 2012;2:e000856. doi:10. 1136/bmjopen-2012-000856

- Prepublication history and additional materials for this paper are available online. To view these files please visit the journal online (http://dx. doi.org/10.1136/ bmjopen-2012-000856).

All presented authors thus fulfil the criteria for authorship.

No else fulfilled the criteria for authorship.

CB and US-M contributed equally to this work.

Received 14 February 2012 Accepted 6 June 2012

This final article is available for use under the terms of the Creative Commons Attribution Non-Commercial 2.0 Licence; see http://bmjopen.bmj.com

For numbered affiliations see end of article.

Correspondence to Professor Ola Hammarsten; ola.hammarsten@clinchem. gu.se

\author{
ABSTRACT \\ Objective: To develop a multimarker prognostic score \\ for infective endocarditis (IE). \\ Design: Retrospective case-control. \\ Setting: Secondary care. Single centre. \\ Participants: 125 patients with definite IE. \\ Primary outcome measures: 90-day and 5-year \\ mortality.
}

Results: Mean age was $62.7 \pm 17$ years. The 90 -day and 5-year mortality was $10.4 \%$ and $33.6 \%$, respectively. CysC levels at admission and over $20 \%$ increases in CysC levels during 2 weeks of treatment were prognostic for 90-day and 5-year mortality independent of creatinine estimated glomerular filtration rate. In multivariate analyses, CysC (OR 5.42, $95 \% \mathrm{Cl} 1.90$ to $15.5, \mathrm{p}=0.002)$ and age (OR $1.06,95 \%$ $\mathrm{Cl} 1.02$ to $1.10, p=0.002$ ) remained prognostic for 5 year mortality. NT-proBNP, TnT, $C$ reactive protein and interleukin 6 were also linked to prognosis. A composite risk scoring system using levels of CysC, NT-proBNP, age and presence of mitral valve insufficiency was able to separate a high-risk and a low-risk group.

Conclusions: CysC levels at admission and increase in CysC after 2 weeks of treatment were independent prognostic markers for both 90-day and 5-year mortality in patients with IE. A multimarker composite risk scoring system including CysC identified a highrisk group.

\section{INTRODUCTION}

Infective endocarditis (IE) is an infection localised to the endocardial surface of the heart. IE mostly involves the heart valves, resulting in local valve destruction and abscess formation as well as development of vegetations with the ability to embolise to various organs. Despite major advances in both diagnostic and therapeutic procedures, neither the incidence ${ }^{1}$ nor the mortality of the disease have decreased in the past 30 years, with a current in-hospital mortality of $15 \%-20 \%$ and 1-year mortality reaching $40 \%$ in developed countries. ${ }^{2}$ Several epide-

\section{ARTICLE SUMMARY}

\section{Article focus \\ - Our aim was to develop a multimarker prognostic score for IE. \\ Key messages \\ - CysC levels at admission and increase in CysC after 2 weeks of treatment were independent prognostic markers for both 90-day and 5-year mortality in patients with IE. \\ - A prognostic score including CysC over $1.2 \mathrm{mg} / \mathrm{l}$, NT-ProBNP over $2000 \mathrm{ng} / \mathrm{l}$, presence of any grade of mitral valve insufficiency (MI) and aged 70 years or older could identify a high- risk and low-risk group in IE. \\ - The prognostic score might be used to improve patient monitoring and assist treatment choices in IE.}

Strengths and limitations of this study

- We were able to monitor changes in levels of biomarkers during treatment in a large cohort of IE patients since blood samples were collected at admission and after 2 weeks of treatment.

- One potential weakness was that $36.2 \%$ of patients treated for IE during the study period (71/196) were unavailable for biomarker studies since they lacked stored blood samples. The mortality was lower in the study group (125) compared with all IE patients treated for IE (196) during the study period.

miological studies have identified a number of prognostic factors related to higher mortality including advanced age, Staphylococcus aureus aetiology, ${ }^{2}{ }^{3}$ cerebral complications $^{4}$ and female sex. ${ }^{5}$ In addition, biomarkers of inflammation like erythrocyte sedimentation rate, ${ }^{2}$ hypoalbuminemia, ${ }^{6}$ leucocytosis, ${ }^{6} \mathrm{C}$ reactive protein $(\mathrm{CRP})^{7}$ and procalcitonin $^{8}$ can predict poor prognosis but are too non-specific to guide therapy in individual patients. Identification of novel prognostic biomarkers and development of a prognostic score could help to identify IE patients who might benefit from more aggressive therapeutic procedures. 
Because IE often influences haemodynamics, biomarkers linked to cardiovascular mortality could have prognostic power in IE. Among these are factors released during cardiovascular stress like NT-proBNP, MR-proANP, copeptin and troponin $\mathrm{T}(\mathrm{TnT})$, which are linked to poor prognosis in heart failure, ${ }^{9}$ coronary syndromes ${ }^{10}$ and sepsis. ${ }^{11}$ Markers of renal function like creatinine, ${ }^{12}$ estimated glomerular filtration rate (eGFR) ${ }^{13}$ and cystatin $\mathrm{C}$ $(\mathrm{CysC})^{14}$ might also be able to predict prognosis in patients with IE similar to their ability to predict cardiovascular mortality. In this study, we analysed clinical factors and cardiovascular biomarkers in blood samples collected at admission and after 2 weeks of therapy among patients with definite IE and examined their ability to predict 90 -day and 5-year mortality. Our primary goal was to develop a prognostic score in IE.

\section{METHODS}

In this single-centre retrospective cohort study, patients with IE treated between 1999 and 2005 at the Department of Infectious Diseases, Sahlgrenska University Hospital, University of Gothenburg, Gothenburg, Sweden, were registered between 1 February 1999 and 3 December 2005. During this time, 196 patients were diagnosed with definite IE according to the modified Duke criteria ${ }^{15}$ and 125 patients had a blood sample drawn at diagnosis and stored and were therefore included in the study. After 2 weeks of treatment, 120 of the 125 patients had a second blood sample drawn. Of all 196 IE patients recorded from 1999 to 2005, 13 patients died $(6.6 \%)$ within 90 days and 43 patients died within 5 years $(20.9 \%)$. Among the 125 patients with an available admission blood sample, the 90-day mortality was $10.4 \%$ and the 5 -year mortality was $33.6 \%$. Information regarding mortality was collected from the hospital's registry of administrative data. The study was approved by the Ethical Committee at the University of Gothenburg. All patients were followed for up to 5 years or until death occurred (mean 1449.1 \pm 636.8 days).

\section{Clinical data}

Demographic and clinical information regarding age, sex, bacterial aetiology, native and prosthetic valve IE, left ventricular ejection fraction (LVEF), comorbidities and surgery during active IE were obtained from the endocarditis database. The study design included analysis of blood samples at admission and after 2 weeks of treatment. The indications for surgery in Sweden are presented elsewhere. ${ }^{16}$

\section{Laboratory analyses}

Serial blood samples were obtained during the hospital stay and stored at $-70^{\circ} \mathrm{C}$ until analysis. The first sample was taken at admission and the second sample 2 weeks later. NT-proBNP was analysed by Elecsys proBNP assay (Roche Diagnostics, Rotkreuz, Switzerland). CysC was analysed in serum using reagents from Dako and the Modular P 2551. Creatinine-based eGFR was calculated using 'The Modification of Diet in Renal Disease' (MDRD) formula. TnT was analysed by using The Elecsys Troponin T high-sensitivity assay (Roche Diagnostics). All biomarkers were analysed on frozen serum samples in a single run. All other laboratory parameters examined were part of the routine laboratory services provided by the Clinical Chemistry Laboratory, Sahlgrenska University Hospital.

\section{Echocardiography}

All patients were examined by transthoracic and transesophageal echocardiography at least once during the study period. Echocardiographic criteria for IE and degrees of valve insufficiency were evaluated. LVEF was calculated from long-axis planes (two-, three- and fourchamber views) of the heart.

\section{Statistical analysis}

Multiple logistic regression models were used to evaluate possible associations between serum levels of biomarkers (NT-proBNP, TnT, creatinine and CysC) and clinical variables including age, sex, echocardiographic parameters and infectious agents and underlying diseases. Univariate comparisons between groups were calculated using conventional t tests. Mann-Whitney $\mathrm{U}$ tests were used for non-parametric comparisons of medians. Dichotomous variables were analysed using the $\chi^{2}$ test. Receiver operating characteristic curves were used to assess the prognostic properties of biomarkers. The logrank test was used to compare different strata in Kaplan-Meier analyses of survival. Statistical analyses were performed with SPSS V.19. All probabilities were two tailed, and $p$ values $<0.05$ were regarded as significant. ORs with CIs were collected from outputs from logistic regression analyses. The coefficient of determination $\left(\mathrm{R}^{2}\right)$ was calculated in Microsoft Excel 2007 using Spearman's correlation to assess the strength of the correlation between CysC change and mortality. The add-in Analyse-it was used to compare AUCs between receiver operating characteristic curves. The study population was chosen to get a power of over $90 \%$ for detecting clinically relevant associations between CysC, NT-proBNP and mortality (expected OR for mortality of at least 2 for each SD increase in the independent variable at a study population of 125 in logistic regression). No missing data existed for the variables included in the prognostic score, but for other tested variables, cases sometimes were excluded if data were missing, although no more than two cases in each analysis had to be excluded due to a high degree of data availability.

\section{RESULTS}

The mean age among the 125 IE patients was $62.7 \pm 16.9$ years, $64.8 \%$ were men and $S$ aureus infection was seen in $28.0 \%$ of the patients (supplementary table 1). Vegetations on aorta, mitralis and tricuspidalis were seen in $50.4 \%, 44.0 \%$ and $4.0 \%$, respectively. Prosthetic valve endocarditis was diagnosed in 28 
(22.4\%) of the patients, and $14(11.2 \%)$ had a pacemaker. Vegetations on pacemaker leads were seen in four of these patients. Most of the patients (91.6\%) had LVEF over $40 \%$, and $34.5 \%$ underwent heart surgery during antibiotic treatment. Clinical parameters correlating with 5-year mortality included age, history of hypertension and mitral valve insufficiency (MI) but not ejection fraction (supplementary table 1). Aminoglycoside use and duration of aminoglycoside therapy were positively correlated with 5-year survival. No associations were found between the presence of emboli and 5-year survival, biomarker levels or clinical variables examined in the study (data not shown).

In the univariate analysis, CysC levels at admission were associated with both 90-day (OR 5.7, 95\% CI 2.2 to $14.7, \mathrm{p} \leq 0.0001)$ and 5-year mortality (OR 7.11, $95 \%$ CI 2.6 to 19.5 , $\mathrm{p} \leq 0.0001$, figure 1 ). CysC increases over $20 \%$ between admission and after 2 weeks of treatment (supplementary figure 1) were also associated with increased 5-year mortality (OR 2.8, 95\% CI 1.20 to 6.6, $\mathrm{p} \leq 0.017)$. The relative and absolute changes of all biomarkers were evaluated, but no significant associations with prognosis were found except for CysC.

All eight patients with CysC over $2.1 \mathrm{mg} / 1$ died within 5 years. CysC levels and increase over $20 \%$ remained significant prognostic indicators for 5-year mortality with similar ORs when alternative multivariate models, including creatinine eGFR or baseline creatinine, were applied (supplementary table 2).

The area under the receiver operator characteristic curve (AUC) for predicting 5-year mortality was 0.70 (95\% CI 0.60 to $0.80, \mathrm{p}<0.001)$ for CysC levels at admission and $0.74(95 \%$ CI 0.65 to $0.83, \mathrm{p}<0.0001)$ for

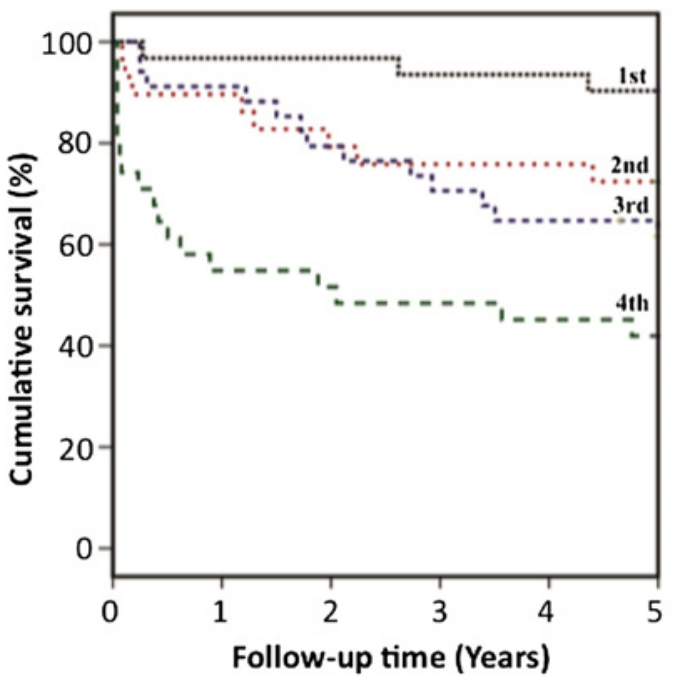

\begin{tabular}{ccccc}
\hline $\begin{array}{c}\text { CysC } \\
\text { quartile }\end{array}$ & $\begin{array}{c}\text { CysC level } \\
(\mathbf{m g} / \mathbf{l})\end{array}$ & $\mathbf{n}$ & $\begin{array}{c}\text { Dead 5 } \\
\text { years (n) }\end{array}$ & p Value \\
\hline 1 & $<0.98$ & 31 & 3 & Reference \\
2 & $0.98-1.17$ & 29 & 8 & 0.066 \\
3 & $1.17-1.52$ & 34 & 13 & 0.008 \\
4 & $>1.52$ & 31 & 18 & $<0.0001$
\end{tabular}

Figure 1 Kaplan-Meier survival plot by quartiles of admission levels of CysC.
CysC levels after 2 weeks of treatment. CysC had a significantly higher AUC compared with creatinine (0.62, 95\% CI 0.51 to $0.73, p=0.0042$ for difference between AUC for CysC and creatinine after 2 weeks of treatment). Mean creatinine was significantly higher among patients who died within 5 years (table 1 ). In contrast, median creatinine was not significantly different among patients who died or lived after 5 years.

In the univariate analysis, log NT-proBNP, GFR, creatinine, age, MI and hypertension were linked to poor prognosis, whereas copeptin and MR-proANP did not reach statistical significance. CRP, interleukin 6 (IL-6) and TnT at 2 weeks, but not at admission, were also linked to prognosis (tables 1 and 2). No sex-based differences were present. There was no association between surgery and prognosis.

The four risk factors with the highest AUC for death within 5 years that appeared to be independent from each other were used to generate a composite risk score where each factor added 1 point. The factors included in the score were CysC over $1.2 \mathrm{mg} / \mathrm{l}$, NT-ProBNP over $2000 \mathrm{ng} / \mathrm{l}$, presence of any grade of MI and aged 70 years or older. The AUC of the risk score was 0.74 (95\% CI 0.70 to $0.87, \mathrm{p}<0.001$ ). If substituting CysC for eGFR calculated from creatinine levels, the AUC decreased to 0.67 (95\% CI 0.57 to 0.78 ).

Patients with a risk score of $0-2$ had a significantly better prognosis $(11 \%(8 / 71)$ died within 5 years $)$ compared with those with a score of 3-4 (63\% (34/54) died within 5 years, $\mathrm{p}<0.0001$ ) (figure 2 ). No significant difference in prognosis could be found between patients differing by 1 point. The score's ability to separate patient prognosis was improved when the $34.5 \%$ of patients that underwent cardiac surgery were excluded (0-2 points $7 \%(3 / 41), 3-4$ points $66 \%(22 / 33)$ died within 5 years, $\mathrm{p}<0.0001)$. The composite risk score also predicted death within 90 -days $(0-2$ points $0 \%(0 / 71)$, 3-4 points $24.1 \%(13 / 54)$ died within 90 days, $\mathrm{p}<0.0001)$.

Furthermore, dividing the cohort based on a score of 0-2 or 3-4 predicted 5-year mortality in patients with left-sided IE (OR 14.6, 95\% CI 5.55 to 38.2 p $<0.001$ ), left-sided IE that did not undergo surgery (OR 25.7, 95\% CI 6.35 to $103.7, \mathrm{p}<0.001)$ and $S$ aureus-infected leftsided IE that did not undergo surgery (OR $31.5,95 \%$ CI 2.35 to $422.3, \mathrm{p}=0.009$ ). Lastly, the score was also able to predict mortality if the MI parameter was excluded (OR $2.30,95 \%$ CI 1.41 to $3.72, \mathrm{p}=0.001$ ) indicating that the composite score was not reliant on the MI parameter and was able to predict mortality in important subgroups of IE.

\section{DISCUSSION}

CysC levels at admission, CysC levels after 2 weeks and over $20 \%$ increases in CysC levels during 2 weeks of treatment were prognostic for mortality in patients with definite IE. The combination of CysC levels and three other risk factors generated a powerful risk score. 
Table 1 Laboratory characteristics of IE patients

\begin{tabular}{|c|c|c|c|c|}
\hline Variable & Total & Dead* & Alive* & p Value \\
\hline CRP (mg/l) (at admission) & $58.6 \pm 57.9$ & $61.1 \pm 54.7$ & $57.3 \pm 64.3$ & 0.72 \\
\hline CRP (mg/l) (after 2 weeks) & $28.0 \pm 49.3$ & $45.0 \pm 26.7$ & $20.7 \pm 78.2$ & 0.013 \\
\hline CRP (mg/l) (peak level) & $139.9 \pm 94.1$ & $160.8 \pm 86.5$ & $130.5 \pm 107.3$ & 0.12 \\
\hline IL-6 (ng/l) (at admission) & $45.3 \pm 112.1$ & $43.5 \pm 134.5$ & $46.3 \pm 42.2$ & 0.90 \\
\hline IL-6 (ng/l) (after 2 weeks) & $24.1 \pm 42.5$ & $39.1 \pm 25.4$ & $17.9 \pm 65.2$ & 0.011 \\
\hline $\mathrm{Hb}(\mathrm{g} / \mathrm{l})$ & $112.2 \pm 17.3$ & $109.8 \pm 17.5$ & $113.2 \pm 16.9$ & 0.48 \\
\hline Creatinine $(\mu \mathrm{mol} / \mathrm{l})$ & $95.3 \pm 66.6$ & $123.2 \pm 26.7$ & $81.4 \pm 104.1$ & $<0.001$ \\
\hline eGFR (ml/min/1.73m²) (MDRD) & $81.0 \pm 41.2$ & $67.3 \pm 44.1$ & $87.0 \pm 29.6$ & 0.016 \\
\hline TnT (ng/l) (at admission) & $106.9 \pm 226.1$ & $156.1 \pm 204.7$ & $82.3 \pm 259.3$ & 0.083 \\
\hline TnT (ng/l) (after 2 weeks) & $75.7 \pm 124.2$ & $109.7 \pm 104.4$ & $61.1 \pm 157.6$ & 0.048 \\
\hline Incremental TnT (\%) & $40.8 \%$ & $44.4 \%$ & $39.3 \%$ & 0.60 \\
\hline NT-proBNP (ng/l) (at admission) & $3874.2 \pm 6868.4$ & $8209.8 \pm 1962.6$ & $1706.4 \pm 10314.3$ & $<0.001$ \\
\hline NT-proBNP (ng/l) (after 2 weeks) & $2597.2 \pm 3363.6$ & $4782.3 \pm 2042.7$ & $1660.8 \pm 4633.1$ & $<0.001$ \\
\hline Incremental NT-proBNP (\%) & $41.7 \%$ & $30.6 \%$ & $46.4 \%$ & 0.11 \\
\hline CysC (mg/l) (at admission) & $1.34 \pm 0.67$ & $1.73 \pm 0.35$ & $1.15 \pm 0.94$ & $<0.001$ \\
\hline CysC (mg/l) (after 2 weeks) & $1.45 \pm 0.81$ & $0.99 \pm 0.65$ & $0.66 \pm 0.99$ & $<0.001$ \\
\hline CysC (>20\% increase) (\%) & $26.7 \%$ & $41.7 \%$ & $20.2 \%$ & 0.015 \\
\hline MR-proANP (pmol/l) (at admission) & $327.6 \pm 199.3$ & $378.8 \pm 227.0$ & $302.0 \pm 452.7$ & 0.21 \\
\hline MR-proANP (pmol/l) (after 2 weeks) & $290.2 \pm 39.6$ & $249.2 \pm 219.1$ & $218.8 \pm 135.1$ & 0.15 \\
\hline Copeptin (pmol/L) (at admission) & $23.3 \pm 29.0$ & $30.1 \pm 24.3$ & $19.9 \pm 59.2$ & 0.17 \\
\hline Copeptin (pmol/L) (after 2 weeks) & $24.0 \pm 29.0$ & $21.2 \pm 31.8$ & $31.7 \pm 21.2$ & 0.42 \\
\hline
\end{tabular}

${ }^{*}$ After 5 years follow-up.

CRP, C reactive protein; eGFR, estimated glomerular filtration rate; IL-6, interleukin 6.

CysC is a small $13 \mathrm{kDa}$ cysteine protease inhibitor produced at a constant rate by all nucleated human cells tested. CysC is cleared predominantly by renal filtration and is most often used as a replacement for creatinine as a marker of GFR. CysC is regarded as a more accurate marker of kidney function than creatinine (reviewed in Lassus and Harjola ${ }^{14}$ ). CysC also offers superior ability to diagnose acute kidney injury ${ }^{17}$ and declining GFR $^{18}$ compared with creatinine. Furthermore, several studies have shown that elevated $\mathrm{CysC}$ is a strong risk factor for adverse cardiovascular prognosis in older people even when creatinine levels are normal. ${ }^{19}$ Increased CysC levels indicate a future risk of developing heart failure, ${ }^{20}$ as well as a poor prognosis among patients with already established heart failure, ${ }^{21}$ independent of creatinine levels. Therefore, CysC is a marker of kidney function and prognosis that outperforms creatinine in most studies.

The associations between CysC levels and prognosis in our study might reflect that IE itself or nephrotoxic agents used during treatment of IE impairs kidney function. The ability of CysC to more correctly predict

Table 2 ORs $^{*}$ for mortality among IE patients

\begin{tabular}{|c|c|c|c|c|}
\hline Variable & OR (all patients) & p Value & OR (no surgery $\ddagger$ ) & p Value \\
\hline Age & $1.06(1.03-1.10)$ & $<0.001$ & $1.09(1.03-1.14)$ & 0.001 \\
\hline MI (all degrees) & $2.94(1.30-6.67)$ & 0.010 & $5.45(1.84-16.2)$ & 0.002 \\
\hline Hypertension & $3.12(1.37-7.10)$ & 0.007 & $2.81(1.03-7.62)$ & 0.043 \\
\hline CRP (mg/l) (after 2 weeks) & $1.01(1.00-1.03)$ & 0.040 & $1.00(0.99-1.01)$ & 0.75 \\
\hline Creatinine $(\mu \mathrm{mol} / \mathrm{l})$ & $1.02(1.00-1.02)$ & 0.006 & $1.02(1.00-1.04)$ & 0.025 \\
\hline GFR (MDRD) & $0.98(0.97-1.00)$ & 0.012 & $0.98(0.96-1.00)$ & 0.041 \\
\hline logNT-proBNP (ng/l) (at admission) & $12.2(4.28-34.9)$ & $<0.001$ & $23.2(4.68-115.0)$ & $<0.001$ \\
\hline logNT-proBNP (ng/l) (after 2 weeks) & $5.91(2.43-14.4)$ & $<0.001$ & $9.44(2.83-31.5)$ & $<0.001$ \\
\hline CysC (mg/l) (at admission) & $7.11(2.59-19.5)$ & $<0.001$ & $37.5(4.58-308.1)$ & 0.001 \\
\hline CysC (mg/l) (after 2 weeks) & $2.55(1.37-4.76)$ & 0.003 & $41.7(5.43-320.6)$ & $<0.001$ \\
\hline CysC (mg/l) (>20\% increase) & $2.82(1.20-6.59)$ & 0.017 & $2.60(0.90-7.50)$ & 0.080 \\
\hline TnT (ng/l) (after 2 weeks) & $1.00(1.00-1.01)$ & 0.059 & $1.03(1.01-1.05)$ & 0.008 \\
\hline IL-6 (ng/l) (after 2 weeks) & $1.02(1.00-1.03)$ & 0.044 & $1.10(1.04-1.17)$ & 0.002 \\
\hline
\end{tabular}

*From multiple logistic regression.

tInsignificant predictors excluded from the table.

$\neq$ Subgroup analysis of patients who did not undergo surgery.

CRP, C reactive protein; GFR, glomerular filtration rate; IL-6, interleukin 6; MI, mitral valve insufficiency. 


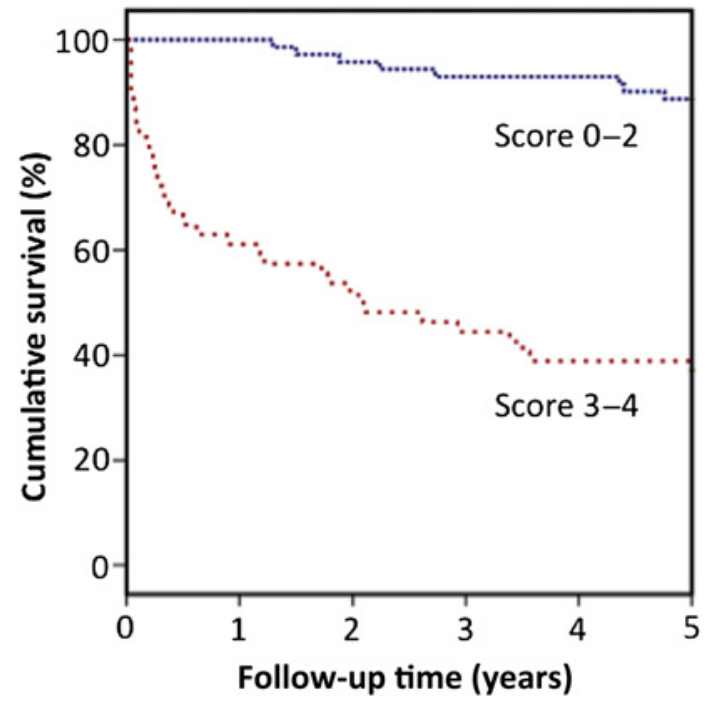

Figure 2 Kaplan-Meier survival plot by risk score for death within 5 years among patients with IE. Patients with $0-2$ points were compared with patients with $3-4$ points $(p<0.001)$. The presence of these risk factors adds 1 point each: any grade of Ml, CysC $>1.2 \mathrm{mg} / \mathrm{l}$, NT-proBNP $>2000 \mathrm{ng} / \mathrm{l}$, aged $>70$ years.

GFR among older people and to respond to rapid changes in GFR compared with creatinine could explain the stronger association between CysC levels and IE prognosis compared with creatinine levels.

There are different conceivable explanations for decreased kidney function in IE. One factor could be decreased cardiac output during the acute phase of IE. Although there was no correlation between LVEF and prognosis (supplementary table 1), levels of the heart failure biomarker, NT-proBNP and prevalence of MI were significantly higher among patients with poor prognosis. In addition, levels of TnT after 2 weeks of treatment were associated with prognosis. TnT levels are affected by cardiac stress found among patients with heart failure $22 \quad 23$ and correlate with prognosis in a number of heart-related conditions. ${ }^{24}$ Therefore, the link between CysC levels and poor prognosis among IE patients could be decreased cardiac output resulting in low kidney perfusion and low GFR.

It is also possible that factors that increase production of CysC, such as increased glucocorticoid levels, ${ }^{25}$ and possibly inflammation, ${ }^{26-28}$ could explain the correlation between CysC levels and IE prognosis. CysC levels show a stronger association with cardiovascular risk factors compared with creatinine, independent of iohexol clearance. ${ }^{29}$ Among patients with chronic renal failure followed for 10 years, CysC levels correlated better with cardiovascular disease mortality compared with creatinine or iothalamate clearance ${ }^{30}$ indicating that CysC levels predict prognosis partly independent from GFR. In line with this possibility, we found that CysC levels and change in CysC levels during 2 weeks of treatment remained prognostic even after multivariate analysis correcting for creatinine levels and creatinine
eGFR (supplementary table 2), suggesting that conditions that increase production of CysC may correlate with IE prognosis. One such factor could be persistent inflammation because high CRP or IL-6 levels after 2 weeks of treatment were also linked to poor prognosis in our study. Moreover, it is also possible that increased glucocorticoid levels due to a general stress response among IE patients could be the link between poor prognosis and CysC levels.

One important aspect of this study was that we could monitor changes in biomarkers during treatment in a large group of IE patients. We found that levels of CRP, IL- 6 and TnT after 2 weeks of treatment, but not admission levels, were significantly associated with 5-year survival. This association likely reflects the fact that a rapid response to initial treatment is of great importance for the prognosis. In contrast, levels of the otherwise promising cardiac stress markers MR-proANP and copeptin failed to predict prognosis, even when levels were assessed after 2 weeks of treatment, indicating that these biomarkers are less valuable among IE patients.

Our results confirmed that MI is a powerful prognostic indicator in IE irrespective of the infected valve. The reason for the significant association between $\mathrm{MI}$ of all degrees, and not with the worst degrees alone (3 and 4), can be explained by the fact that the patients with severe MI often underwent surgery, which is linked to improved survival. $^{31}$ This association could, however, not be confirmed in this study (supplementary table 1).

The multitude of biomarkers and clinical factors included in this study allowed us to explore a composite risk score based on the four strongest and apparently independent risk factors. The composite risk score was able to separate a high-risk and a low-risk group with significantly different 90-day and 5-year survival. IE has still a poor prognosis, despite major advances in diagnostic and therapeutic procedures, and we still have limited ability to find the patients that should be considered for more aggressive treatment. In this light, this novel risk score could add decisional information and allow for a multifactorial judgement of patients and consideration for more active intervention like heart valve replacement or surgical removal of vegetations.

A potential problem with the current study was that $36.2 \%$ of the patients treated for IE during the study period $(71 / 196)$ lacked stored blood samples. In addition, there was a bias for patients with a worse prognosis in the study group. On the other hand, the study group of 125 IE patients had a 5-year prognosis $(66.4 \% 5$-year survival) closer to the $60 \%$-year survival reported in most studies ${ }^{1}{ }^{2}$ compared with the complete cohort (79\% 5-year survival) indicating that the prognosis in our study group were comparable with the outcome in previous studies. Furthermore, data on haemodynamic parameters on admission and during the hospital stay were not recorded. As this was a single-centre study, our findings must be validated before the risk score can be included in clinical routine. 
In summary, a prognostic score including CysC over $1.2 \mathrm{mg} / \mathrm{l}$, NT-proBNP over $2000 \mathrm{ng} / \mathrm{l}$, presence of any grade of MI and aged 70 years or older could identify a high-risk and low-risk group in IE.

\section{Author affiliations}

${ }^{1}$ Department of Medicine, Sahlgrenska University Hospital, The Sahlgrenska Academy at University of Gothenburg, Gothenburg, Sweden

${ }^{2}$ Department of Infectious diseases, Sahlgrenska University Hospital, The Sahlgrenska Academy at University of Gothenburg, Gothenburg, Sweden ${ }^{3}$ Department of Clinical Chemistry and Transfusion Medicine, Sahlgrenska University Hospital, The Sahlgrenska Academy at University of Gothenburg, Gothenburg, Sweden

Acknowledgements We are thankful for the expertise and analysis of the laboratory parameters by Bodil Gustafsson, Carina M Gustafsson and Anne-Sofie Johansson Fällgren.

Contributors All authors have contributed significantly to the conception and design of the manuscript, interpretation of data, drafting the article and revisions for important intellectual content and final approval of the version to be published. CB, US-M and LO have also worked on the acquisition and analysis of data. All authors have approved the final version of the manuscript submitted.

Funding This work was supported by the Swedish Cancer Society $(\mathrm{OH})$, Swedish Research Council $(\mathrm{OH})$, Swedish Pain Foundation $(\mathrm{OH})$, the Sahlgrenska University Hospital Research Foundation (MLXF, OH), Swedish Heart-Lung Foundation (MLXF), Sahlgrenska University Hospital Research Foundation (MLXF) and by government support to the city councils for cost arising (grant ALFGBG-138141) (L0).

Competing interests None.

Ethics approval Ethical approval was provided by the Ethical Committee at the University of Gothenburg.

Provenance and peer review Not commissioned; externally peer reviewed.

Data sharing statement There are no additional data available.

\section{REFERENCES}

1. Tleyjeh IM, Steckelberg JM, Murad HS, et al. Temporal trends in infective endocarditis: a population-based study in Olmsted County, Minnesota. JAMA 2005;293:3022-8.

2. Murdoch DR, Corey GR, Hoen B, et al. Clinical presentation, etiology, and outcome of infective endocarditis in the 21st century: the International Collaboration On Endocarditis-Prospective Cohort Study. Arch Intern Med 2009;169:463-73.

3. Thuny F, Di Salvo G, Belliard O, et al. Risk of embolism and death in infective endocarditis: prognostic value of echocardiography: a prospective multicenter study. Circulation 2005;112:69-75.

4. Thuny F, Avierinos JF, Tribouilloy C, et al. Impact of cerebrovascular complications on mortality and neurologic outcome during infective endocarditis: a prospective multicentre study. Eur Heart $J$ 2007;28:1155-61.

5. Sambola A, Fernandez-Hidalgo N, Almirante B, et al. Sex differences in native-valve infective endocarditis in a single tertiary-care hospital. Am J Cardiol 2010;106:92-8.

6. Wallace SM, Walton $\mathrm{BI}$, Kharbanda RK, et al. Mortality from infective endocarditis: clinical predictors of outcome. Heart 2002;88:53-60.

7. Olaison L, Hogevik H, Alestig K. Fever, C-reactive protein, and other acute-phase reactants during treatment of infective endocarditis. Arch Intern Med 1997;157:885-92.

8. Kocazeybek B, Küçükoğlu S, Oner YA. Procalcitonin and C-reactive protein in infective endocarditis: correlation with etiology and prognosis. Chemotherapy 2003;49:76-84.

9. Palazzuoli A, Gallotta M, Quatrini I, et al. Natriuretic peptides (bnp and nt-probnp): measurement and relevance in heart failure. Vasc Health Risk Manag 2010;6:411-18.
10. Mazzone M, Forte $\mathrm{P}$, Portale $\mathrm{G}$, et al. Brain natriuretic peptide and acute coronary syndrome. Minerva Med 2005;96:11-18.

11. Perman SM, Chang AM, Hollander JE, et al. Relationship between Btype natriuretic peptide and adverse outcome in patients with clinica evidence of sepsis presenting to the emergency department. Acad Emerg Med 2011;18:219-22.

12. Rostagno C, Rosso G, Puggelli F, et al. Active infective endocarditis: clinical characteristics and factors related to hospital mortality. Cardio J 2010;17:566-73.

13. Hillege $H$, Van Gilst $W$, de Zeeuw D, et al. Renal function as a predictor of prognosis in chronic heart failure. Heart Fail Monit 2002;2:78-84.

14. Lassus J, Harjola VP. Cystatin C: a step forward in assessing kidney function and cardiovascular risk. Heart Fail Rev 2012;17:251-61.

15. Li JS, Sexton DJ, Mick N, et al. Proposed modifications to the Duke criteria for the diagnosis of infective endocarditis. Clin Infect Dis 2000;30:633-8

16. Westling K, Aufwerber E, Ekdahl C, et al. Swedish guidelines for diagnosis and treatment of infective endocarditis. Scand J Infect Dis 2007;39:929-46.

17. Nejat M, Pickering JW, Walker RJ, et al. Rapid detection of acute kidney injury by plasma cystatin $\mathrm{C}$ in the intensive care unit. Nephrol Dial Transplant 2010;25:3283-9.

18. Premaratne E, Maclsaac RJ, Finch S, et al. Serial measurements of cystatin $\mathrm{C}$ are more accurate than creatinine-based methods in detecting declining renal function in type 1 diabetes. Diabetes Care 2008;31:971-3.

19. Ix JH, Shlipak MG, Chertow GM, et al. Association of cystatin C with mortality, cardiovascular events, and incident heart failure among persons with coronary heart disease: data from the Heart and Soul Study. Circulation 2007;115:173-9.

20. Sarnak MJ, Katz R, Stehman-Breen CO, et al. Cystatin C concentration as a risk factor for heart failure in older adults. Ann Intern Med 2005;142:497-505.

21. Alehagen U, Dahlström U, Lindahl TL. Cystatin $\mathrm{C}$ and NT-proBNP, a powerful combination of biomarkers for predicting cardiovascular mortality in elderly patients with heart failure: results from a 10-year study in primary care. Eur $J$ Heart Fail 2009;11:354-60.

22. Peacock WF 4th, De Marco T, Fonarow GC, et al. Cardiac troponin and outcome in acute heart failure. $N$ Engl $J$ Med 2008;358:2117-26.

23. deFilippi CR, de Lemos JA, Christenson $\mathrm{RH}$, et al. Association of serial measures of cardiac troponin $T$ using a sensitive assay with incident heart failure and cardiovascular mortality in older adults. JAMA 2010;304:2494-502.

24. Omland T, de Lemos JA, Sabatine MS, et al. A sensitive cardiac troponin T assay in stable coronary artery disease. $N$ Engl J Med 2009;361:2538-47.

25. Bökenkamp A, van Wijk JA, Lentze MJ, et al. Effect of corticosteroid therapy on serum cystatin $\mathrm{C}$ and beta2-microglobulin concentrations. Clin Chem 2002;48:1123-6.

26. Evangelopoulos AA, Vallianou NG, Bountziouka V, et al. Association between serum cystatin $\mathrm{C}$, monocytes and other inflammatory markers. Intern Med J 2012;42:517-22.

27. Grubb A, Björk J, Nyman U, et al. Cystatin C, a marker for successfu aging and glomerular filtration rate, is not influenced by inflammation. Scand J Clin Lab Invest 2011;71:145-9.

28. Stevens LA, Schmid CH, Greene T, et al. Factors other than glomerular filtration rate affect serum cystatin $\mathrm{C}$ levels. Kidney Int 2009;75:652-60.

29. Mathisen UD, Melsom T, Ingebretsen OC, et al. Estimated GFR associates with cardiovascular risk factors independently of measured GFR. J Am Soc Nephrol 2011;22:927-37.

30. Menon V, Shlipak MG, Wang X, et al. Cystatin C as a risk factor for outcomes in chronic kidney disease. Ann Intern Med 2007; 147:19-27.

31. Lalani T, Cabell $\mathrm{CH}$, Benjamin DK, et al; International Collaboration on Endocarditis-Prospective Cohort Study (ICE-PCS) Investigators. Analysis of the impact of early surgery on in-hospital mortality of native valve endocarditis: use of propensity score and instrumental variable methods to adjust for treatment-selection bias. Circulation 2010;121:1005-13. 